\title{
Survey of Medical Oncology Status in Korea (SOMOS-K): A National Survey of Medical Oncologists in the Korean Association for Clinical Oncology (KACO)
}

\author{
Do Yeun Kim, MD, MPH, PhD' \\ Yun Gyoo Lee, MD² \\ Bong-Seog Kim, MD, PhD 3
}

${ }^{1}$ Department of Internal Medicine, Dongguk University Ilsan Hospital, Goyang, ${ }^{2}$ Department of Internal Medicine, Kangbuk Samsung Hospital, Seoul, ${ }^{3}$ Department of Internal Medicine, Veterans Health Service Medical Center, Seoul, Korea
Correspondence: Bong-Seog Kim, MD, PhD Department of Internal Medicine, Veterans Health Service Medical Center, 53, Jinwhangdoro 61-gil, Gangdong-gu, Seoul 05368, Korea

Tel: 82-2-2225-1319

Fax: 82-2-2225-4149

E-mail: seog@bohun.or.kr

Received July 16, 2016

Accepted August 29, 2016

Published Online September 23, 2016

\section{Purpose}

This study was conducted to investigate the current role of medical oncologists in cancer care with a focus on increasing the recognition of medical oncology as an independent specialty.

\section{Materials and Methods}

Questionnaires modified from the Medical Oncology Status in Europe Survey dealing with oncology structure, resources, research, and patterns of care given by medical oncologists were selected. Several modifications were made to the questionnaire after feedback from the insurance and policy committee of the Korean Association for Clinical Oncology (KACO). The online survey was then sent to KACO members.

\section{Results}

A total of 214 medical oncologists (45.8\% of the total inquiries), including 71 directors of medical oncology institutions, took the survey. Most institutions had various resources, including a medical oncology department (94.1\%) and a department of radiation oncology (82.4\%). There was an average of four medical oncologists at each institution. Medical oncologists were involved in various treatments from diagnosis to end-of-life care. They were also chemotherapy providers from a wide range of institutions that treated many types of solid cancers. In addition, $86.2 \%$ of the institutions conducted research.

\section{Conclusion}

This is the first national survey in Korea to show that medical oncologists are involved in a wide range of cancer treatments and care. This survey emphasizes the contributions and proper roles of medical oncologists in the evolving health care environment in Korea.

\section{Introduction}

Medical oncologists are core members of multidisciplinary teams that mediate the evidence-based, safe, and cost-effective use of cancer drugs while preserving the quality of life of patients. A recent position paper by the European Society for Medical Oncology (ESMO) emphasized the role of medical oncologists in the care of cancer patients in clinical and translational research, and it described the active political
Key words

Medical oncology, Surveys and questionnaires, Korea involvement of medical oncologists $[1,2]$.

The ESMO published two reports on the status of medical oncology based on the results of the Medical Oncology Status in Europe Survey (MOSES) [3,4]. The study described the status of medical oncology in Europe, which mainly involved teaching the discipline to undergraduate students and postmedical graduates, and a multidisciplinary approach to cancer treatment. Phase III MOSES stated that medical oncology could be improved by providing a greater number of medical oncology courses to undergraduate students and greater 
postgraduate specialization and/or subspecialization, although this differed between countries. The roles of medical oncologists in overall care and multidisciplinary collaboration were emphasized and showed improvement over time, while organ specialists still played a significant role.

In Korea, medical oncology has been recognized as a separate specialty since 1997, and 504 physicians were boardcertified in medical oncology as of July 2015 [5]. Cancer treatment is becoming more specialized and complex; therefore, the need for multidisciplinary approaches to cancer treatment is increasing. The Korean Association for Clinical Oncology (KACO) was established in 2005 to enhance knowledge and facilitate multidisciplinary care for cancer patients. Although medical oncologists have played an increasing role in cancer patient care in Korea, their current status in clinical practice has not been adequately evaluated.

Therefore, this survey was conducted to provide an overall analysis of the status of medical oncologists and to improve the recognition of medical oncology as an independent specialty in Korea. This is the first national survey of medical oncologists accredited by the KACO.

\section{Materials and Methods}

This was a prospective study evaluating the current status of medical oncologists in Korea. Questionnaires modified from the MOSES were first reviewed by select representatives of the insurance and policy committee of the KACO. Feedback and reviews were exchanged, and 53 questions to be answered by directors of departments of medical oncology and 27 questions to be answered by individuals working at each institution were finally selected. The major components included are listed below.

(1) General information: demographics and an overview of medical oncology facilities; (2) clinical practice: the types of multidisciplinary collaborations and the proportion of work performed by medical oncologists; (3) clinical research: the numbers of clinical studies performed and protocols proposed to the Korean Cancer Study Group (KCSG).

Questions involving an overview of facilities, the pattern of multidisciplinary collaboration, and clinical research were answered by the director of each institution. We used an electronic questionnaire. The online survey was sent to full members of the KACO in August 2015 via email, and those who did not respond were sent follow-up emails requesting responses by October 2015. Our local Institutional Review Board approved this study (Dongguk University Ilsan Hospital protocol number 2015-104).

\section{Results}

A total of 214 of 467 individuals (45.8\%) who were sent the survey completed it. Overall, $60 \%$ of the directors of departments of medical oncology replied (71/119), while $41 \%$ of individuals replied (143/348). The answers from the directors of medical oncology departments were as follows.

\section{General information}

\section{1) Characteristics}

Males comprised $59 \%$ of the respondents, with a mean age of 42 years (range, 29 to 70 years). Overall, 98\% reported using chemotherapy in clinical practice. Most responses were sent by individuals in Seoul and the surrounding metropolitan area $(64 \%)$.

\section{2) Overview of medical oncology facilities}

Overall, $47.7 \%$ of surveyed institutions $(32 / 68)$ were cancer centers. The distribution of facilities related to medical oncology is shown in Fig. 1. Most institutions were equipped with a department of medical oncology $(94.1 \%)$ and a department of radiation oncology (82.4\%). A clinical trial center and palliative care center were present in less than $50 \%$ of the institutions.

The mean number of medical oncologists in an institution was four (range, 1 to 21). Specialists who were fully responsible for palliative care were present in $32.4 \%$ of the institutions (22/68). Subspecialties of medical oncologists were present at 16 institutions, and family medical doctors at six institutions.

\section{Clinical practice}

\section{1) Percentages of multidisciplinary collaborations for the four major types of solid cancers}

Fig. 2 shows the average percentage of multidisciplinary collaborations at the institutions for the four major solid cancers (lung cancer, breast cancer, stomach cancer, and colorectal cancer). Although multidisciplinary care was provided from diagnosis to end-of-life, the percentage of multidisciplinary care was less than $10 \%$. Major decisions were made via multidisciplinary collaboration in the following proportion of cases: lung cancer, $26.6 \%$; breast cancer, $18.3 \%$; stomach cancer, $21 \%$; and colorectal cancer, $30.2 \%$. 


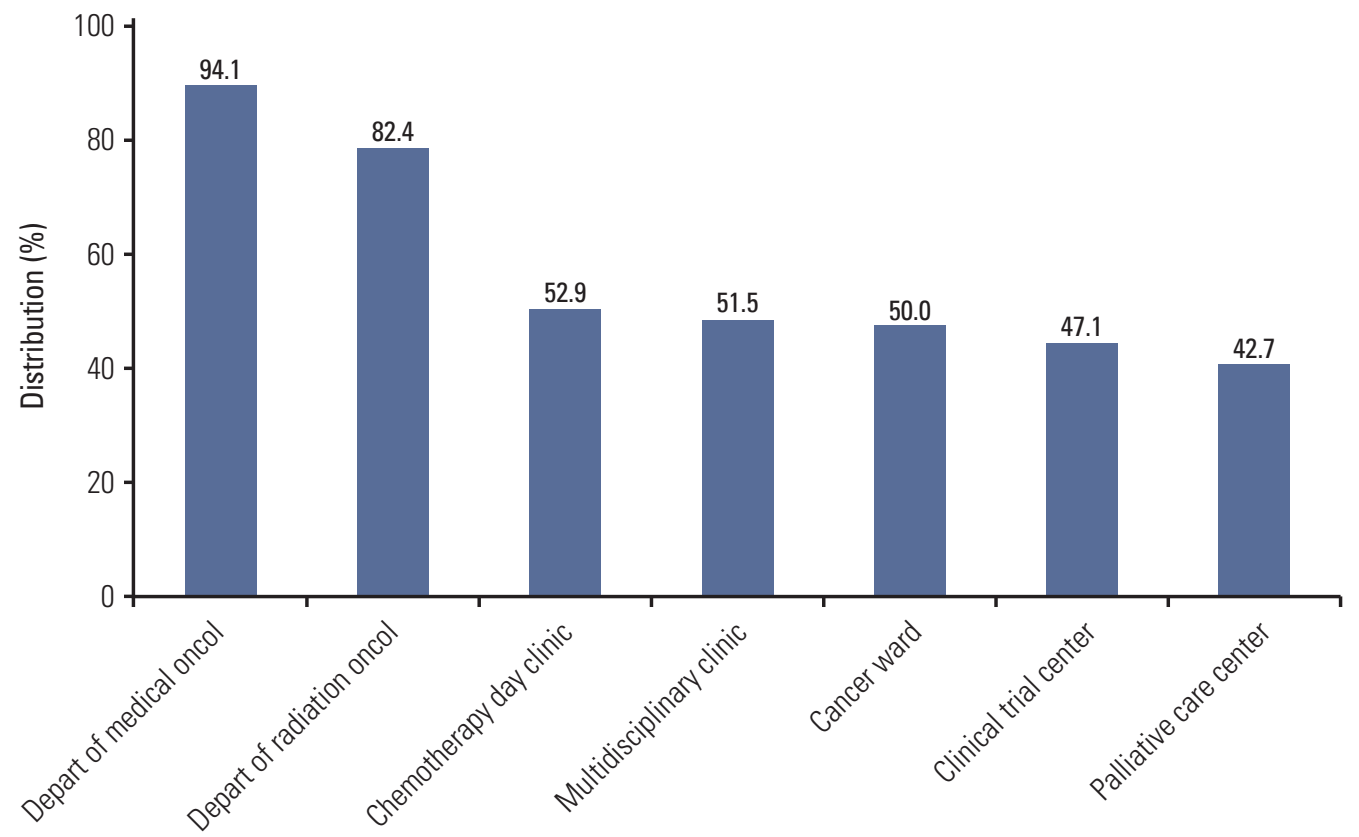

Fig. 1. Distribution of medical oncology facilities at institutions $(n=68)$. Depart of medical oncol, Department of Medical Oncology; Depart of radiation oncol, Department of Radiation Oncology.

\section{2) The role of medical oncologists in patient care for the four major solid cancers}

Fig. 3 shows the average percentages of medical oncologists involved in treating the four major solid cancers. Organ specialists (e.g., surgical oncologists, pulmonologists, and gastroenterologists) were mainly involved in diagnoses. Medical oncologists contributed significantly to the administration of chemotherapy, which was the most common palliative chemotherapy given to patients with stomach cancer $(84.5 \%)$, while it was the least common adjuvant chemotherapy for breast cancer (33.3\%). Medical oncologists provided adjuvant chemotherapy in cases of lung cancer (50\%), stomach cancer (50\%), and colorectal cancer (45.8\%), and were involved in palliative care/end-of-life care in approximately $60.0 \%-77.6 \%$ of the cases.

\section{3) The role of medical oncologists as chemotherapy providers in the treatment of other solid cancers}

Among the 62 respondents, the average percentage of medical oncologists who were chemotherapy providers was $42.0 \%$ for central nervous system cancer, $84.0 \%$ for head and neck cancer, $41.9 \%$ for hepatobiliary cancer, and $44.0 \%$ for genitourinary cancer.

\section{Research}

Overall, $86.2 \%$ of institutions were performing clinical research.

\section{1) Research facilities}

An institutional review board was present at all 71 institutions. There were clinical research nurses, clinical trial centers, animal laboratories, and cellular laboratories at $84.0 \%$, $59.0 \%, 59.0 \%$, and $57.1 \%$ of the institutions, respectively.

\section{2) Number of clinical trials performed}

We surveyed the number of clinical trials performed during the previous year (Fig. 4). More than 10 clinical trials were conducted at 11 institutions $(11 / 49,22.4 \%)$ involved in global trials, and seven institutions involved in domestic trials $(7 / 53,13.2 \%)$. A range of one to five of the institutions $(44.7 \%)$ performed sponsor-initiated clinical trials (SITs), while $71.2 \%$ conducted investigator-initiated clinical trials (IITs). Phase I, phase II, and phase III trials were performed at $40.6 \%, 80 \%$, and $83.3 \%$ of the institutions, respectively. Most (37/56, 66.1\%) of the institutions participated in clinical trials involving the KCSG. 
A
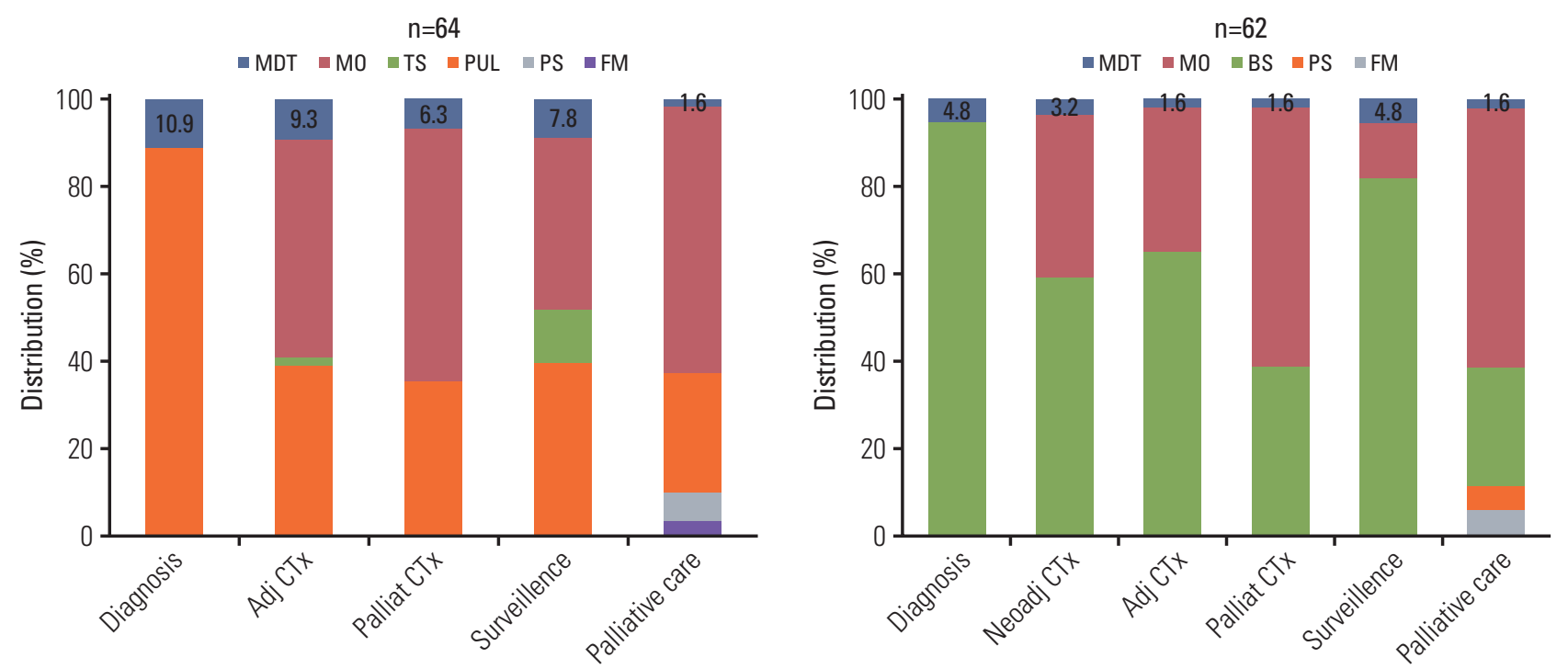

C
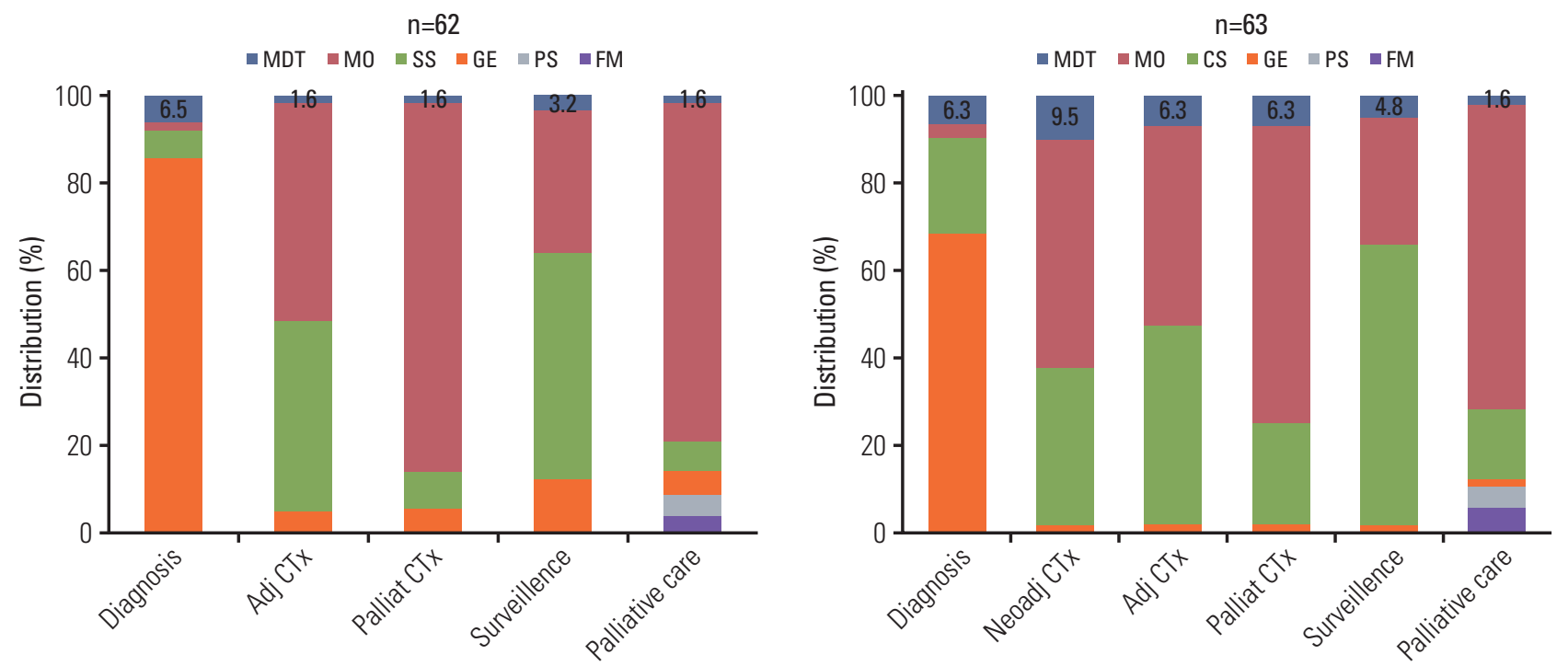

Fig. 2. The average percentage of multidisciplinary cancer care at institutions that provided care for the four major cancer types of cancer: lung cancer (A), breast cancer (B), stomach cancer (C), and colorectal cancer (D). Adj CTx, adjuvant chemotherapy; Palliat CTx, palliative chemotherapy; Neoadj CTx, neoadjuvant chemotherapy; MDT, multidisciplinary team; $\mathrm{MO}$, medical oncologist; TS, thoracic surgeon; PUL, pulmonologist; PS, palliative care specialist; FM, family medicine doctor; BS, breast surgeon; SS, stomach surgeon; GE, gastroenterologist; CS, colorectal surgeon.

\section{Discussion}

This is the first survey to conduct an overall analysis of the current status of medical oncologists in Korea. The results showed that medical oncologists are involved in various phases of cancer treatment to different degrees, and that they actively contribute to cancer research.

As of 2015, 504 physicians were accredited with a specialty in medical oncology and / or hematology in Korea. The status of medical oncologists differs between countries. We analyzed the status of medical oncologists in Korea using a European survey (MOSES) prepared by the ESMO MOSES Task Force in 2008 [4]. The survey measured items such as 

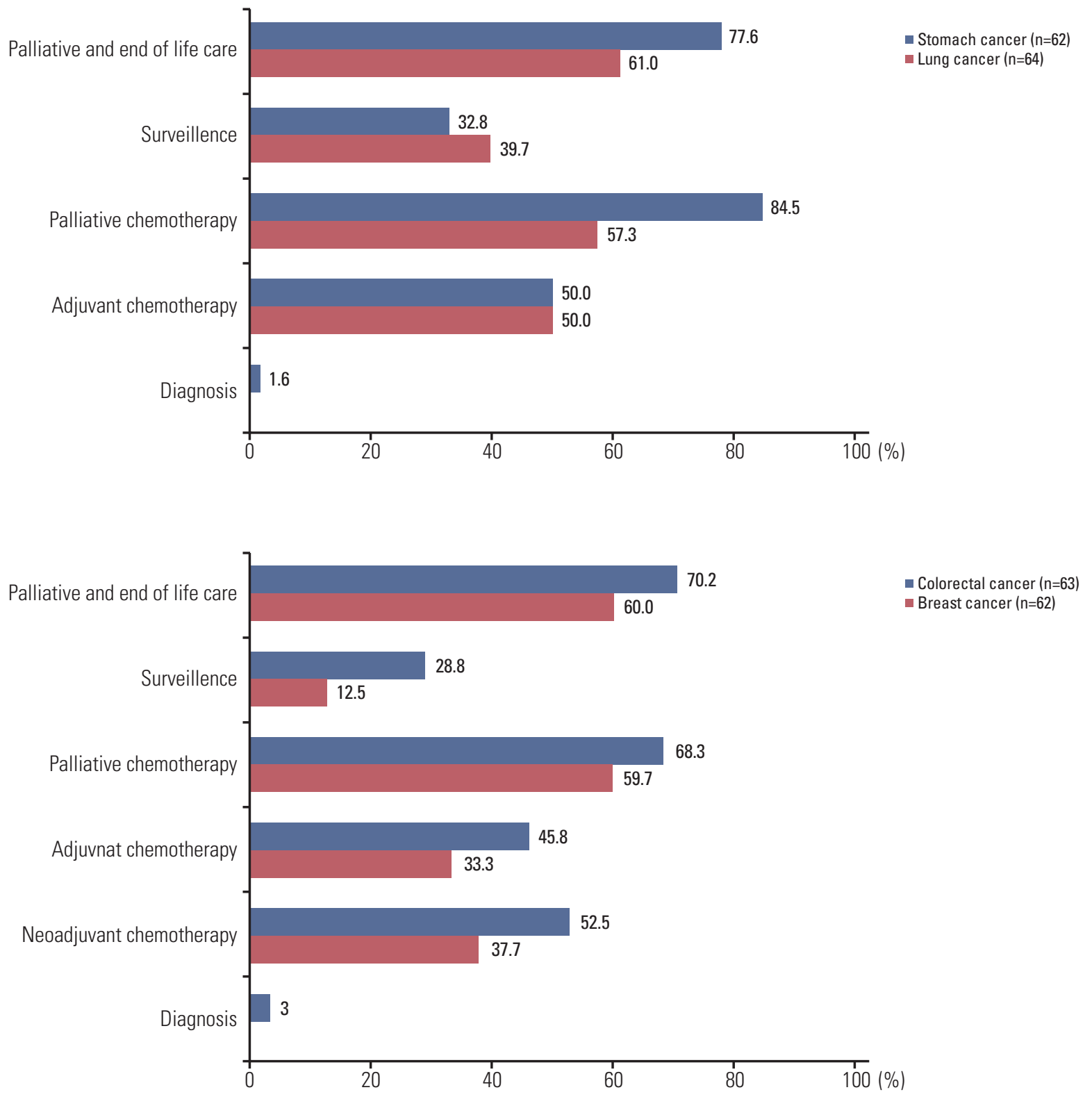

Fig. 3. Average percentage of medical oncologists involved in the care of cancer patients with the four major types of solid cancers.

the teaching of oncology to undergraduate students, postgraduate specialization and/or subspecialization in oncology, continuing medical education, national internal certification, oncology facilities, types of cancer care, multidisciplinary collaboration, and prescription/administration of cytotoxic therapy. The current study, Survey of Medical Oncology Status in Korea (SOMOS-K), mainly asked questions regarding clinical practice.

MOSES III comprehensively reported the types of cancer care and multidisciplinary collaboration [4]. The results showed that the role of medical oncologists in multidisciplinary teams is important and improving over time. However, organ specialists (e.g., gastroenterologists, pulmonologists, urologists, and gynecologists) also played an important role, consistent with our findings. Because there are few data describing the percentage of medical oncologists that contribute to the treatment of various types of cancer, it is not possible to compare our data directly with those from other national medical societies, and this percentage is most likely dependent on each nation's health care infrastructure, health 


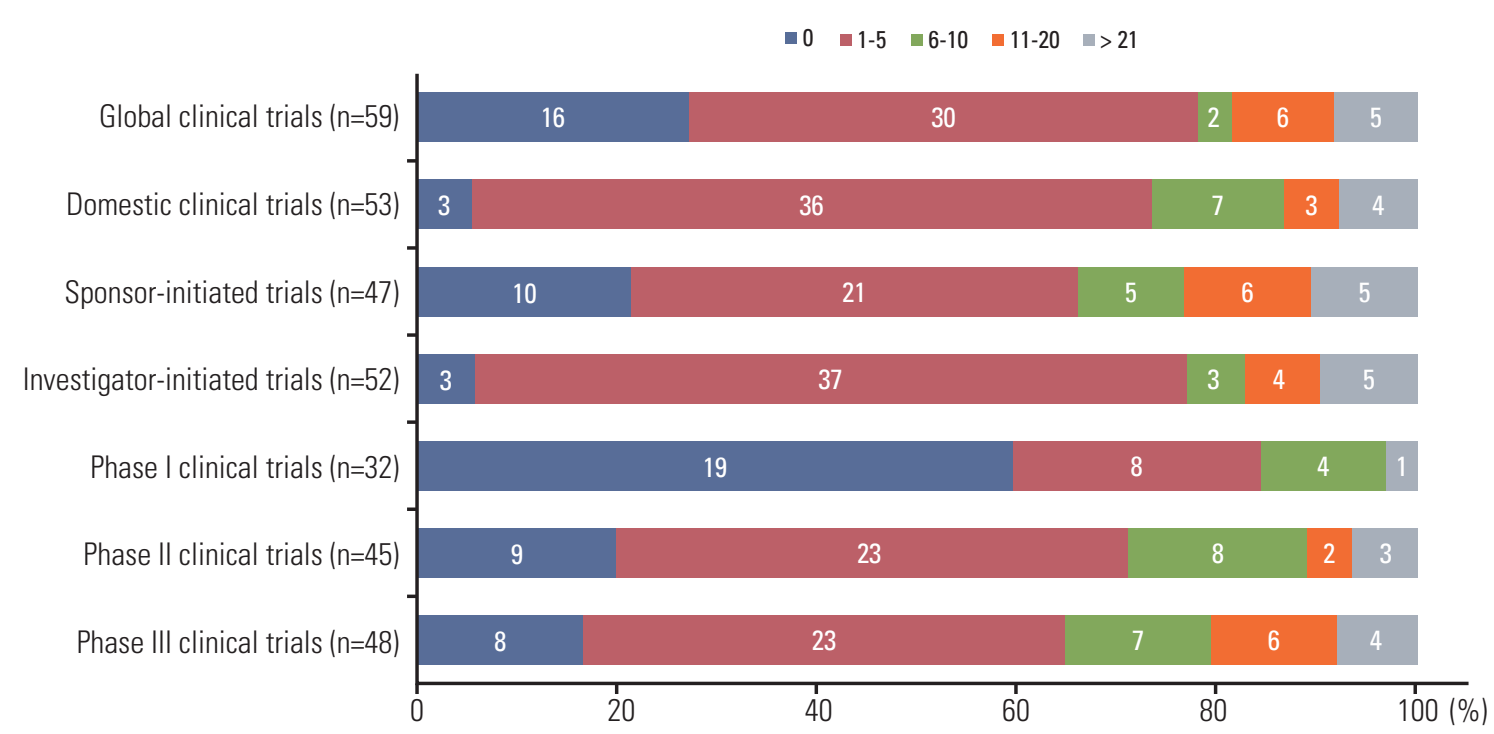

Fig. 4. The number of clinical trials in Korea conducted within the past year at institutions.

policies, and the number of active oncology professionals. According to MOSES III, nonmedical oncology professionals were mainly involved in hormone-related therapies. Moreover, European cancer patients experienced more difficulties receiving reimbursement for treatments prescribed by specialists other than medical oncologists because of legal issues regarding the handling and administration of cytotoxic drugs. Korea should emphasize the position of the ESMO, which states that medical oncologists are cancer specialists trained to provide treatment with drugs, ranging from chemotherapy to newly targeted agents and immunotherapies. That paper also stated that only specialized medical oncology training could prepare physicians to provide optimal care because of the increasingly complex scientific advances [2].

We found that clinical cancer trials are actively conducted in Korea, and that an impressive number of Korean medical oncologists are involved in global cancer clinical trials, with 5 institutions engaged in more than 20 global clinical trials. In addition, the number of IITs was higher than that of SITs, and five institutions (5/47 SITs, $10.6 \%$ and 5/52 IITs, 9.6\%) performed more than 10 clinical trial SITs and / or IITs during the previous year. Furthermore, a recent analysis of clinical cancer trials in Korea reported that the number of such trials is rapidly increasing [6]. The authors suggested that the rapid growth of IITs is due to increased interest in clinical trials by Korean physicians, and the effects of multicenter clinical trial organizations such as the KCSG. However, they also mentioned the lack of funding for IITs, as well as a need for more financial support from government agencies and public donations. Nevertheless, medical oncologists should actively establish their roles as clinical researchers in this innovative area of cancer medicine.

The political involvement of medical oncologists also included anticancer drug approval, reimbursement systems, participation in risk-benefit assessments of new drugs, and plans for potential future workforce shortages. The medical oncologist workforce was another important issue. Although the United Kingdom recommended 1.25 whole time equivalent medical oncologists $/ 200,000-250,000$ persons in 2000 , there are no international recommendations regarding the optimal proportion of medical oncologists for the general population [7]. Accordingly, increasing numbers of studies of the workforce necessary to fulfill the needs of cancer services are being conducted. For example, the American Society of Clinical Oncology began a workforce survey about 10 years ago and recently reported a projected shortage of oncologists (overall demand will grow by $40 \%$, whereas supply will grow by only $25 \%$ ) by the year 2025 . A rapidly aging population and increasing medical insurance were considered main factors in this discrepancy [8,9]. Although there are no exact numbers regarding the medical oncologist workforce in Korea, there is no reason to suspect that the situation in Korea is different. The World Health Organization also has concerns about the workforce available to meet the need for cancer services. Korean medical oncologists, including the KACO group, must therefore be proactive in solving this problem $[10,11]$.

It should be noted that our study has some limitations. First, questionnaires are inherently not fully reliable. Second, 
the percentage of medical oncologists involved in cancer care and as chemotherapy providers was based primarily on the reports of directors at each institution. Thus, this information may be insufficient to determine the current status of medical oncologists in Korea. Further investigation using data extracted from health insurance records and assessments of records from other government agencies that deal with health policies will be necessary to fully characterize the roles of medical oncologists in the treatment of cancer.

\section{Conclusion}

The data presented by the SOMOS-K should be used to enhance the contribution of medical oncologists to cancer care and to emphasize the vital contributions that medical oncologists make to the currently evolving health care environment.

\section{Conflicts of Interest}

Conflict of interest relevant to this article was not reported.

\section{Acknowledgments}

This survey was supported by the KACO and presented in part at the 10th annual meeting of the KACO (2015). The authors express their appreciation to Professor Hyun-Cheol Chung, President of the KACO, and to all other members of the KACO for participating in the questionnaire and their valuable support.

\section{References}

1. Popescu RA, Schafer R, Califano R, Eckert R, Coleman R, Douillard JY, et al. The current and future role of the medical oncologist in the professional care for cancer patients: a position paper by the European Society for Medical Oncology (ESMO). Ann Oncol. 2014;25:9-15.

2. The ASCO POST January 15, 2014. ESMO releases position paper on med-oncs' role in cancer care [Internet]. Huntington, NY: HSP News Service; 2016 [cited 2016 Jul 7]. Available from: http: // www.ascopost.com/issues/ january-15-2014/ esmoreleases-position-paper-on-med-oncs-role-in-cancer-care.aspx.

3. The ESCO MOSES Task Force. Medical Oncology Status in European Survey (MOSES) Phase II [Internet]. ViganelloLugano: ESMO; 2016 [cited 2016 Jul 7]. Available from: https:// www.esmo.org/ content/ download / 8359/170041/ file/2006-MOSES.pdf.

4. The ESCO MOSES Task Force. Medical Oncology Status in European Survey (MOSES) Phase III [Internet]. ViganelloLugano: European Society for Medical Oncology; 2016 [cited 2016 Jul 7]. Available from: https:// www.esmo.org/content/ download / 8358 / 170037 / file / 2008-ESMO-MOSES-PhaseIII. pdf.

5. The Korean Association of Internal Medicine. Sixth education for resident training staff. Seoul: The Korean Association of Internal Medicine; 2015. p. 47-8.

6. Shim BY, Park SH, Lee S, Kim JS, Lee KE, Kang YK, et al. Current status and challenges of cancer clinical trials in Korea.
Cancer Res Treat. 2016;48:20-7.

7. Centre for Workforce Intelligence (CFWI). Medical Oncology [Internet]. Centre for Workforce Intelligence (CFWI); 2016 [cited 2016 Jul 7]. Available from: http://www.cfwi.org.uk/ publications / medical-oncology-cfwi-medical-fact-sheet-andsummary-sheet-august-2010/attachment.pdf.

8. Erikson C, Salsberg E, Forte G, Bruinooge S, Goldstein M. Future supply and demand for oncologists: challenges to assuring access to oncology services. J Oncol Pract. 2007;3: 79-86.

9. Yang W, Williams JH, Hogan PF, Bruinooge SS, Rodriguez GI, Kosty MP, et al. Projected supply of and demand for oncologists and radiation oncologists through 2025: an aging, betterinsured population will result in shortage. J Oncol Pract. 2014;10:39-45.

10. European Society for Medical Oncology. Experience sharing by other societies who have performed national workforce surveys. ESMO global cancer task force project of the ESMO emerging countries committee. Viganello-Lugano: European Society for Medical Oncology; 2015.

11. European Society for Medical Oncology. WHO of a proposal for a workforce survey to answer the question: is the projected workforce able to meet the need to deliver cancer services? ESMO global cancer task force project of the ESMO emerging countries committee. Viganello-Lugano: European Society for Medical Oncology; 2015. 